\title{
ARTICLE \\ Associations among peripheral and central kynurenine pathway metabolites and inflammation in depression
}

\author{
Ebrahim Haroon (D) ${ }^{1}$, James R. Welle ${ }^{2}$, Bobbi J. Woolwine ${ }^{1}$, David R. Goldsmith ${ }^{1}$, Wendy Baer ${ }^{1}$, Trusharth Patel ${ }^{3}$, Jennifer C. Felger ${ }^{1}$ and \\ Andrew H. Miller ${ }^{1}$
}

\begin{abstract}
Kynurenine pathway (KP) metabolites are believed to be a link between inflammation and depression through effects on brain glutamate receptors. However, neither the relationship between plasma and cerebrospinal fluid (CSF) KP metabolites nor their association with inflammatory mediators is well-established in depression. Moreover, the clinical profile associated with combined activation of plasma inflammatory and kynurenine pathways is unknown. Accordingly, plasma and CSF-KP metabolites and inflammatory markers along with depressive symptoms and antidepressant treatment response were measured in 72 unmedicated depressed patients. Following bivariate analyses, component factors representing immune and kynurenine variables in the plasma and CSF were extracted and were used to examine directionality of associations in a path model. In addition, patients were clustered using individual markers that most accounted for the association between plasma immune and KP systems. Path analysis revealed a directional association extending from plasma inflammatory markers to plasma kynurenines, to CSF kynurenines. Among immune markers, plasma tumor necrosis factor (TNF) was robustly associated with plasma kynurenine (KYN) and KYN/tryptophan (TRP), which was in turn significantly associated with CSF KYN, kynurenic acid, and quinolinic acid. Clustering of patients based on plasma TNF and KYN/TRP yielded subgroups of high $(N=17)$ and low $(N=55)$ TNF-KYN/TRP groups. High TNF-KYN/TRP subjects exhibited greater depression severity, anhedonia, and treatment nonresponse. In conclusion, plasma-KP metabolites may mediate an inflammation-associated depressive symptom profile via CNS KP metabolites that can serve as a target for intervention at the level of inflammation, peripheral KYN metabolism, KYN transport to the brain, or effects of KP metabolites on glutamate receptors.
\end{abstract}

Neuropsychopharmacology (2020) 45:998-1007; https://doi.org/10.1038/s41386-020-0607-1

\section{INTRODUCTION}

There is a growing appreciation of the potential role of the kynurenine pathway (KP) in the relationship between inflammation and depression. Inflammatory cytokines such as tumor necrosis factor (TNF) and interferon- $\gamma$ (IFN- $\gamma$ ), as well as peripherally administered inflammatory stimuli such as lipopolysaccharide (LPS) and Bacillus Calmette-Guérin (BCG) vaccine induce the KP by activating the enzyme indoleamine-2,3 dioxygenase (IDO), which converts tryptophan (TRP) to kynurenine (KYN) [1-3]. Based on studies in laboratory animals, KYN then accesses the brain through the large neutral amino acid transporter (LAT-1) and is subsequently metabolized to kynurenic acid (KYNA) in astrocytes, oligodendrocytes, and neurons or 3-hydroxy kynurenine (3HKYN), anthranilic acid (AA), 3-hydroxy anthranilic acid (3HAA), and ultimately quinolinic acid (QA) in microglia, tissue macrophages, and perivascular monocytes [4-8]. A critical target for the two neuroactive end products of the KP is the $N$-methyl-D-aspartate (NMDA)-glutamate receptor with QA acting as an agonist and KYNA acting as an allosteric modulator at the glycine site [9-11]. Blocking KP activation in mice through inhibition of IDO reverses LPS- and BCG-induced depressive-like behavior [3, 12]. Moreover, blocking the NMDA receptor reduces LPS-induced depressive-like behavior [13]. In humans, increases in KP metabolites in the plasma and cerebrospinal fluid (CSF) have been associated with both the onset of depression and greater depression severity in medically ill patients receiving the inflammatory cytokine IFN-a [14, 15]. Moreover, an increased plasma KYN/TRP ratio was associated with greater volume loss in the striatum among depressed but not control subjects and the KYNA/QA ratio showed a trend association with anhedonia in depressed patients $[16,17]$. Increased KYN was also significantly associated with reduced motivation in elderly individuals [18]. In addition, CSF QA was elevated acutely after a suicide attempt and remained persistently elevated during a 2-year follow-up period [19-21]. Finally, although inflammation has been associated with a poor response to conventional antidepressants [22], decreases in the KYN/TRP predicted response to the novel antidepressant ketamine [23].

The influence of KYNs on the pathophysiology of depression has focused on their role in the central nervous system (CNS) [24]. However, in studies in rodents, central concentrations of KP metabolites appear to be determined by KYN metabolism in the periphery. For example, the competitive blockade of KYN passage into the brain by saturating LAT-1 with an excess of leucine reduces LPS-induced depressive-like behavior in mice [25]. Thus, targeting the KP to intervene in depression in humans requires an understanding of the complex interplay between KP metabolites and inflammatory mediators in the periphery and the brain. Accordingly, we examined the associations between KP

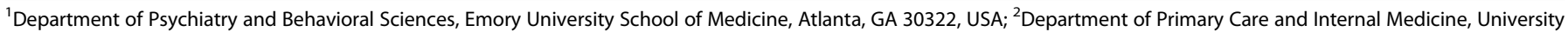
of Colorado School of Medicine, Denver, CO 80045, USA and ${ }^{3}$ Department of Anesthesiology, Emory University School of Medicine, Atlanta, GA 30322, USA

Correspondence: Ebrahim Haroon (eharoon@emory.edu)

Received: 6 November 2019 Revised: 23 December 2019 Accepted: 30 December 2019

Published online: 15 January 2020 
metabolites in the plasma and CSF (a proxy for brain levels), and their associations with inflammatory mediators in these compartments. Based on the preclinical data, we hypothesized that positive direct associations would exist between plasma inflammatory mediators and plasma-KP metabolites (Plasma-KP) than, in turn, would demonstrate similar associations with CSF-KP metabolites (CSF-KP). We further hypothesized an indirect association between plasma immune and CSF-KP mediated by Plasma-KP. We also hypothesized that combined plasma inflammatory and KP activation would identify a subgroup of depressed subjects with greater severity of depression, anhedonia, and treatment nonresponse.

\section{MATERIALS AND METHODS}

\section{Participants}

Seventy-two subjects aged 21-65 years with a major depressive episode based on Structured Clinical Interview for Diagnostic and Statistical Manual-IV (SCID-IV) [26] and a 17-Item Hamilton Rating Scale for Depression score $(H A M-D) \geq 18$ [27] were recruited from newspaper, radio, television, and social media campaigns advertising for depressed individuals not taking psychiatric medications. All subjects were medically stable as determined by medical history, physical exam, and laboratory testing. Exclusion criteria included evidence of an autoimmune or inflammatory disorder, or infectious disease (Hepatitis B, C, or human immunodeficiency virus); use of an immunosuppressant drug (e.g., oral glucocorticoids) within the past 6 months or regular use of a nonsteroidal anti-inflammatory drug or statins; active suicidal ideation; history of a psychotic disorder of any type, mania, or mixed state, or substance abuse/dependence within the past 6 months (determined by SCID-IV and urine drug testing). Due to potential effects of psychotropic medications on the immune response [28], all subjects were free of antidepressants, antipsychotics, mood stabilizers, and regular use of benzodiazepines for at least 4 weeks ( 8 weeks for fluoxetine) prior to study participation. No subject was removed from psychotropic medication for the purposes of this study, and all patients were carefully monitored by study staff for worsening of depression or the development of suicidal ideation during study participation. As dictated by their primary care physicians, subjects could continue medications for any medical disorders including $81 \mathrm{mg}$ per day of aspirin. All subjects provided written informed consent and the study was approved by the Institutional Review Board of Emory University. Subjects represent a subgroup of patients recruited for a study on phenotyping depression with increased inflammation (NCT\#01426997) that have been included in previous reports [22, 29-32].

\section{Blood and CSF sampling}

Participation in this study involved a 2 day stay in the inpatient research unit at Emory University Hospital with blood sampling occurring on Day 1 and CSF sampling (lumbar puncture) on Day 2. Blood sampling and the lumbar puncture were conducted within $24 \mathrm{~h}$ of each other but were performed on separate days to avoid the impact of the stress of lumbar puncture on blood assay results. Blood was obtained through an indwelling catheter between 0900 and $1100 \mathrm{~h}$ after $30 \mathrm{~min}$ of rest to minimize circadian variations and the stress of venipuncture. Blood was immediately centrifuged at $1000 \times g$ for $10 \mathrm{~min}$ at $4^{\circ} \mathrm{C}$ and plasma was collected and stored at $-80^{\circ} \mathrm{C}$ for batched assays. Lumbar puncture was performed between 1200 and $1600 \mathrm{~h}$ by an anesthesiologist. CSF samples were aliquoted and stored at $-80^{\circ} \mathrm{C}$ until batched assays.

Immune and KYN metabolite assays

Plasma concentrations of high-sensitivity c-reactive protein (CRP) were determined by immunoturbidometric method (Sekisui Diagnostics, Lexington, MA, USA) on a Beckman AU480 automatic analyzer and CSF CRP was quantified by electrochemiluminescence-based immunoassay (MesoScale Discovery, Gaithersburg, MD). Plasma and CSF TNF, soluble TNF receptor 2 (TNFR2), interleukin-1 $\beta$ (IL1b), IL1 receptor antagonist, IL6, IL6 soluble receptor, IL10, and monocyte chemoattractant protein-1 were assayed in duplicate (plasma) or triplicate (CSF) using multiplex bead-based assays (R\&D Systems, Minneapolis, MN, USA) and read with a MAGPIX charge-coupling device imaging system (Luminex, Austin, TX) as previously described [31]. Mean coefficients of variation (CVs) for all immune assay techniques were reliably $<10 \%$ and no samples were below the limits of detection. KP metabolites were assayed in one batch to avoid batch-to-batch variability. Plasma and CSF concentrations of TRP, KYN, KYNA, 3HKYN, AA, 3HAA, and QA were measured using high-performance liquid chromatography and tandem mass spectrometry in a commercial laboratory (formerly Brains Online, LLC, now Charles River, Inc.) as previously described [17]. Mean assay CVs were reliably $<7 \%$ and the lower limit of quantification (LLOQ) in plasma and CSF are provided in Supplementary Table S1. Due to multiple measurements below the LLOQ, 3HAA in the CSF was not included in statistical analyses. The number of subjects with missing values for other immune and KP metabolites, as well as behavioral measures (see below), were minimal and are detailed in Supplementary Table S2.

Measures of depression severity, anhedonia, and treatment response

The 30-item Inventory for the Severity of Depression (Self-Report Version, IDS-SR) [33] was conducted on Day 2 in the morning before the lumbar puncture and was used to assess overall depressive symptom severity and symptom clusters for the purposes of statistical analyses. Items assessing appetite (\#11-12), weight (\#13-14), and diurnal variation (\#9A-9B) were excluded from the total IDS-SR score as suggested in scoring guidelines [33]. Given the previous literature linking severity of anhedonia with immune and KP metabolites, summary scores of anhedonia-specific items on the IDS-SR scale (\#21-Capacity for Pleasure or Enjoyment, \#20-Energy Level, \#19-General Interest, and \#8-Response of Your Mood to Good or Desired Events) identified in previous studies were combined to yield summary anhedonia scores [34]. The sum of individual scores of the four anhedonia items selected from IDS-SR combined into a subscale with a reliability Cronbach's $a=0.73$. Antidepressant treatment was assessed using Massachusetts General HospitalAntidepressant Treatment Response Questionnaire (MGH-ATRQ) based on the number and adequacy of antidepressant treatment trials ( $>6$ weeks at the recommended dosage) during the current episode of depression [22, 35]. MGH-ATRQ scores were collapsed into three groups namely 0 (score $=0$, no previous trials), 1 (scores $=1-3$ ), and 2 (scores $>3$ ), with an increasing score from 0 to 3 , indicating greater treatment resistance. Previous research has indicated that MGH-ATRQ scores $>3$ may signify underlying treatment resistance [36-38]. Subjects were not recruited based on history of treatment response (or nonresponse). Many patients $(n=42)$ had received no medication treatment during their current episode. As actively suicidal patients were ineligible for study participation and assessment of suicidal ideation/behavior was available only as a single item on the IDS-SR, suicide was not considered in the analyses.

\section{Statistical analysis}

Measures of immune and KP markers were quantile-normalized to minimize variations induced by both sample source (blood and CSF) and technical heterogeneity, and to yield distributions with identical statistical properties necessary for the proposed statistical approaches $[39,40]$. Furthermore, the normality of transformed data was confirmed using Shapiro-Wilk's test [41]. Linear associations were examined using Pearson's correlations. Confirmatory factor 
analysis (CFA) was then used to extract four summary factor scores representing plasma- and CSF-KP, and -immune measures using previously published guidelines [42-44]. To minimize errors and improve fit, only markers with significant loadings on their component factors were included. The hypothesized directionality of associations between the four summary factor scores were examined using a mediational path analysis. Mediational path analysis separates total linear effects into direct (attributed to the variable of interest) and indirect effects (induced via another intermediary variable) as described previously $[45,46]$. To identify which individual immune marker best accounted for the plasma-KP system as reflected by KYN/TRP (indexing peripheral IDO activity), variable selection using least absolute shrinkage and selection (Lasso)/elasticnet-based methods was used. Lasso/elasticnet methods complemented by Bayesian variable selection were employed to minimize the impact of the large number of intercorrelations among immune variables including linear, quadratic, and interactive effects $[47,48]$. Linear/nonlinear association between the identified plasma immune marker and KYN/TRP ratio was then examined using partial and pieced regressions, and the residuals were examined for clustering effects. Iterative $k$-means clustering was used to identify a subsample with combined elevations of the identified immune marker and KYN/TRP ratio. Lasso inferential models were used to examine associations between group status (identified above) and clinical severity measures after partialing out the effects of covariates with $k(10)$-fold repetitions resampled $=\# 10$ (100-fold repeats). Age, sex, ethnicity (Caucasian vs. African American), and body mass index were included as prespecified covariates in all models, given their previously demonstrated associations with both immune and KP metabolites [16, 49-51]. To minimize overfitting and reduce prediction error, cross-validation was performed using randomly split Discovery (training) and Validation (holdout) sets $(n=36$ each). Finally, the reproducibility of the relationships between group status and clinical variables were compared using area under the curve (AUC) generated from receiver operating curves. A priori thresholds of practical significance based on published guidelines [52] were used with a sample size of 72, an $a$ of 0.05 , and $(1-\beta)$ of 0.8 , and effect size of Cohen's $d=0.8$ or its linear $\left(r^{2}=0.20\right)$ or nonlinear equivalents $(A \cup C=0.7)$. Detailed description of the analysis plan and methods are provided in Supplementary Materials and Methods, and the working model and analytic pipeline is presented in Supplementary Fig. S1. In line with previous publications, plasma KYN/TRP ratios were multiplied by 1000 to facilitate graphical presentation [14].

\section{RESULTS}

\section{Sample}

Seventy-two subjects with both blood and CSF samples were included in the analyses. Table 1 provides data on clinical and selected biomarker variables that survived correction for false discovery rate. Descriptive statistics on all available metabolites are presented in Supplementary Table S3.

\section{Bivariate associations between plasma- and CSF-KP and immune} measures

Multivariate associations between plasma- and CSF-KP, as well as their association with plasma and CSF immune measures are depicted as a correlation heatmap in Fig. 1. KP metabolites in the plasma strongly correlated with each other (all $p<0.01$ ), whereas several KP metabolites in the plasma strongly correlated with KP metabolites in the CSF. Plasma KYN/TRP was similarly robustly associated with CSF KYN/TRP, KYN, AA, KYNA, and QA (Pearson's $r=$ $0.77,0.72,0.60$, and 0.52 , respectively (all $p<0.0001$; Fig. 2a-d). In addition, significant correlations were found between plasma and CSF AA, as well as between plasma and CSF QA (both $p<0.0001$ ). Plasma 3HKYN was also significantly associated CSF QA $(p<0.0001)$. Of the plasma immune markers, plasma TNF and TNFR2 were strongly associated with KYN and QA in the plasma, whereas plasma 3HKYN was correlated only with plasma TNFR2 (all $p<0.0001$ ). Supplementary Table S4 lists all pairwise correlations that survived Bonferroni correction.

Extraction of component factor scores and path analysis Four summary factor scores representing Plasma-KP, CSF-KP, plasma immune (Plasma-IMM) and CSF immune (CSF-IMM) measures were extracted using CFA. All factor models demonstrated robust goodness-of-fit, and high item reliability (see Supplementary Table S5A-C). The extracted factors were entered into a non-recursive path model to examine the hypothesized directionality of effects from Plasma-IMM to Plasma-KP to CSF-KP. Sex was excluded, as it was unrelated to the extracted factors. Paths linking Plasma-IMM to Plasma-KP $\left(X^{2}(1)=10.69, p=0.001\right)$, Plasma-KP to CSF-KP $\left(X^{2}(1)=10.86, p=0.001\right)$, and Plasma-IMM to Plasma-KP to CSF-KP $\left(X^{2}(1)=12.74, p<0.001\right)$ were all significantly stronger than the path from CSF-IMM to CSF-KP. Strong direct effects were noted between Plasma-IMM and Plasma-KP, and between Plasma-KP to CSF-KP $(p<0.001)$, and the indirect association between Plasma-IMM and CSF-KP was exclusively mediated by Plasma-KP $(p<0.001)$. Figure 3 provides a graphic summary of standardized direct, indirect, and total effects, and Supplementary Table S6A-D provide detailed analytic information. An overall power $=0.9$ for the path model was obtained using estimated effect sizes (Cohen's $f=0.43$ and 0.82 , partial $r^{2}=0.33$ and 0.45 for Plasma- and CSF-KP factors, respectively) with tested/ total predictors $=1 / 7$ (including covariates), $a=0.007(0.05 / 7)$, and sample size $=72$ (Supplementary Fig. S2).

Clustering of subjects based on Plasma immune markers and KYN/ TRP

Plasma KYN/TRP (indexing IDO activity) was used as the dependent variable to represent the KP system because of its robust correlations with other KP metabolites in the plasma and CSF (see above), and its extensive use in the literature [8, 14, 15, 18]. Moreover, plasma KYN/TRP could be combined with plasma immune markers to identify putative targets for biomarker profiling. Plasma immune markers were entered as independent variables and prespecified covariates as control factors in the Lasso-selection and related Bayesian variable selection-based Lasso models. The quadratic term of plasma TNF was selected as the strongest predictor of plasma KYN/TRP by the consensus panel of all 9/9 lasso/ elasticnet models and Bayesian-Lasso models (Supplementary Table S7A, B).

Comparison of the linear and quadratic fit of the relationship between plasma TNF and KYN/TRP (Fig. 4a) indicated that the curved (quadratic/polynomial) fit was significantly stronger than the linear fit (linear/nonlinear slope difference $[F(1,69)=11.3, p=0.001]$. A pieced regression splitting the quadratic slope into two independent regression lines above/below plasma TNF $=5.67 \mathrm{pg} /$ $\mathrm{ml}$ indicated that although intercepts were not different between both levels $[F(1,68)=1.38, p=0.24]$; the slope for TNF $>5.67 \mathrm{pg} / \mathrm{ml}$ was greater than both the null $=0[\mathrm{~F}(1,68)=48.24, p<0.001]$ and compared with $\mathrm{TNF}<5.67 \mathrm{pg} / \mathrm{ml}$ slope $[\mathrm{F}(1,68)=11.11, p=0.001]$. On the contrary, TNF $<5.67 \mathrm{pg} / \mathrm{ml}$ slope was insignificant $[\mathrm{F}(1,68)=$ $0.86, p=0.36]$ and not different from the null. This possibility of clustering between the two markers was further confirmed by examinations of the actual-by-predicted and residual-by-predicted plots. These findings supported the use of clustering approaches using TNF and KYN/TRP. Based on iterative $k$-means clustering with cluster sizes ranging from 2 to 12 and based on cubic clustering criteria (CCC), a two-group solution with optimal $C C C=1.95$ was chosen to partition subjects into two groups: a "high" TNF-KYN/TRP group $(n=17)$ and a "low" TNF-KYN/TRP group $(n=55)$. Figure $4 \mathrm{~b}$ illustrates clustering of plasma TNF and KYN/TRP in high and low TNF-KYN/TRP groups using a connected scatterplot and Table 1 provides a contrast of clinical and biological variables between 
Table 1. Summary table of key background variables and biomarkers.

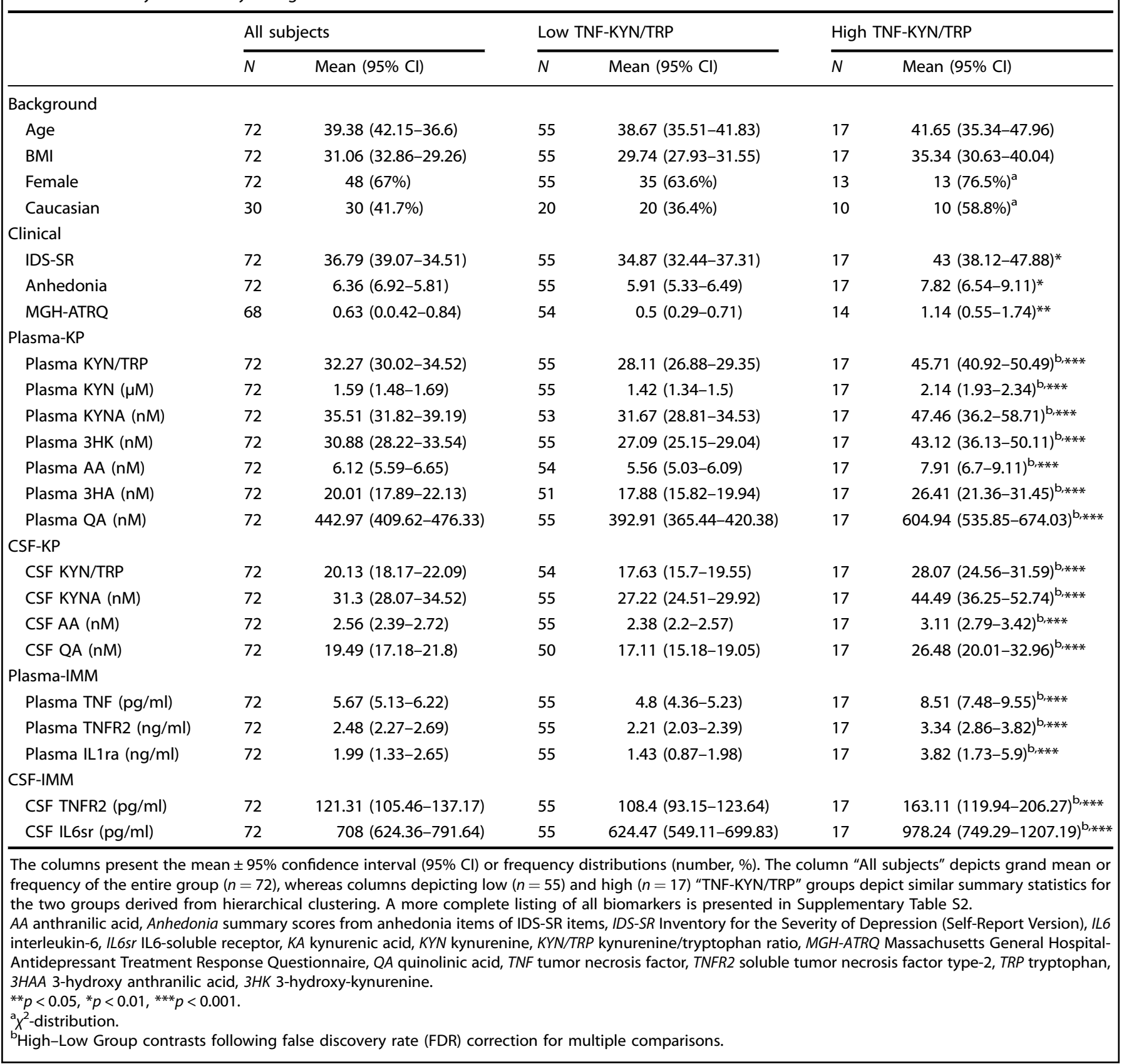

groups. Details of cluster separation and validation are provided in Supplementary Table S8 and Supplementary Fig. S3.

Plasma TNF-KYN/TRP and depression severity

High TNF-KYN/TRP group status (Wald's $z=1.91, p=0.05$ ) explained IDS-SR scores better than plasma TNF $(z=-3.93$, $p=0.22)$ or $\mathrm{KYN} / \mathrm{TRP}$ ratio $(z=0.15, p=0.95)$ alone or the TNF*KYN/TRP interaction term $(z=1.93, p=0.45)$. High TNFKYN/TRP status continued to be strongly associated with IDS-SR scores (Wald's $z=3.21, p=0.001$ ) in an independent cross-fit lasso inferential model. The 8.17-point IDS-SR score difference $(95 \% \mathrm{Cl}=3.18-13.2)$ seen in the high vs. low TNF-KYN/TRP group contrast exhibited a strong effect size $d(95 \% \quad \mathrm{Cl})=0.90$ (0.34-1.47). A power analysis comparing $r^{2}$-values of the full model (four covariates + TNF-KYN/TRP $=0.22$ ) with that from the reduced model (only TNF-KYN/TRP grouping $=0.13$ ) yielded a power of $(1-\beta)$ of 0.81 for the current samples size of 72 (Supplementary Fig. S4).

TNF-KYN/TRP and anhedonia severity

High TNF-KYN/TRP group was associated with greater anhedonia scores on the anhedonia subscale (Wald's $z=2.88, p=0.004$ ). The 1.89-point anhedonia score difference $(95 \% \mathrm{Cl}=0.60-3.17)$ seen in the high vs. low TNF-KYN/TRP group contrast had a strong effect size $d(95 \% \mathrm{Cl})=0.85(0.29-1.41)$. A power analysis using $r^{2}$ comparison between the full $(0.24)$ and reduced $(0.12)$ model indicated a power of 0.91 with the current sample size of 72 (Supplementary Fig. S4).

TNF-KYN/TRP and antidepressant treatment response After partialing out covariates, the high TNF-KYN/TRP group was also significantly associated with greater MGH-ATRQ scores 


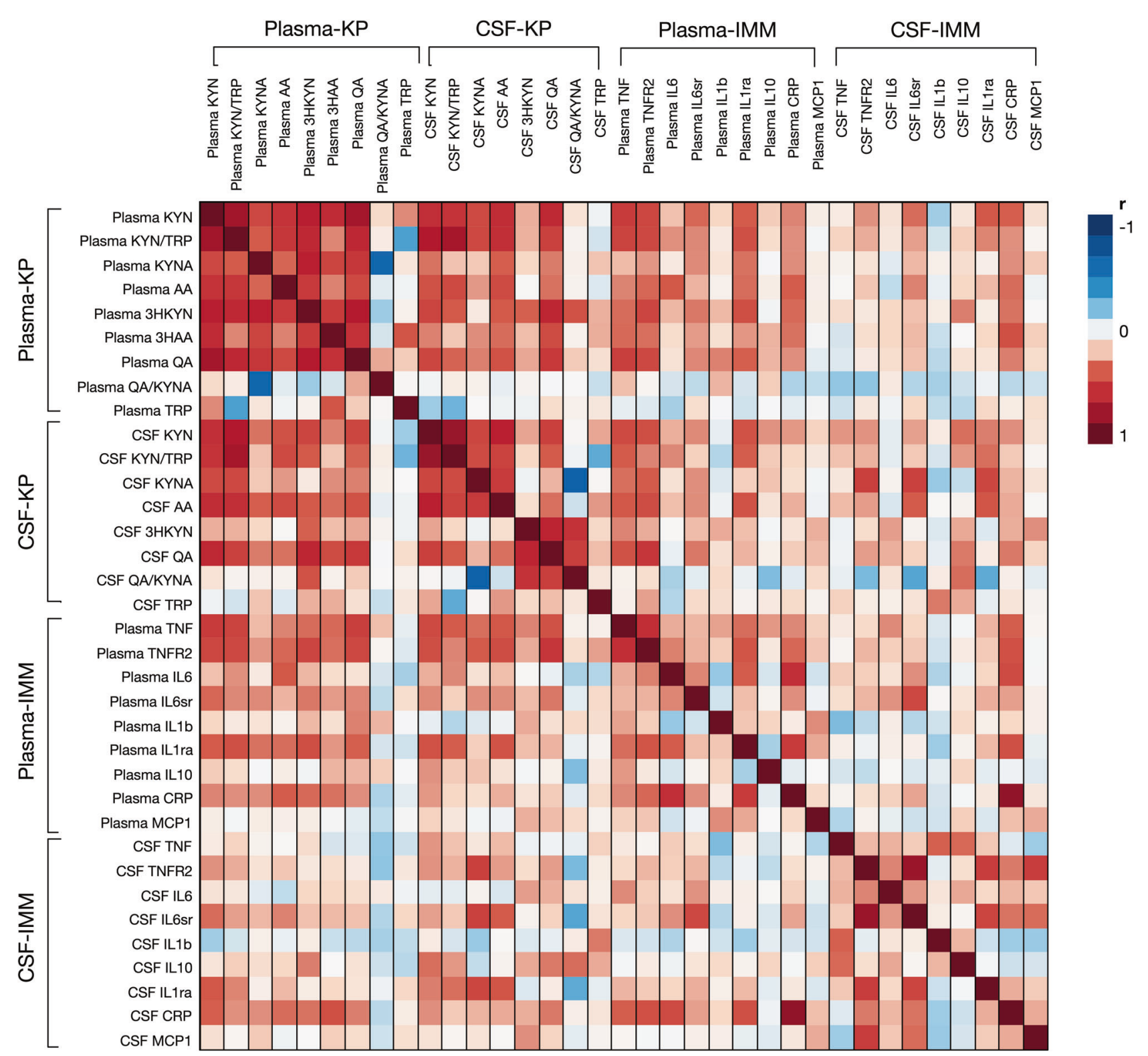

Fig. 1 Correlation heatmap of immune and kynurenine pathway markers. The $x$ - and $y$-axes represent quantile-normalized kynurenine and immune markers in the plasma and cerebrospinal fluid (CSF) compartments, and accompanying $r$-scale represents strength and directionality of Pearson's product-moment correlation coefficients. Bonferroni-corrected $p$-values of the above correlation coefficients are provided in Supplementary Table S4. Abbreviations: AA anthranilic acid, CRP c-reactive protein, IL6 interleukin-6, IL6sr IL6-soluble receptor, IL10 interleukin-10, IL1b interleukin-1 $\beta$, IL1ra IL1b-receptor antagonist, KYN kynurenine, KYN/TRP kynurenine/tryptophan, KYNA kynurenic acid, MCP1 monocyte chemoattractant protein-1, QA quinolinic acid, QA/KYNA quinolinic acid/kynurenic acid, TNF tumor necrosis factor, TNFR2 soluble tumor necrosis factor type-2, TRP tryptophan, 3HAA 3-hydroxy anthranilic acid, 3HKYN 3-hydroxy kynurenine.

(Wald's $z=2.59, p=0.009$ ). Post hoc testing indicated that the corrected mean difference in MGH-ATRQ between groups was $0.63(95 \% \mathrm{Cl}$ difference $=(0.07-1.19)$, which yielded an effect size of $d(95 \% \mathrm{Cl})=0.78(0.18-1.39)$. Comparisons of full $\left(r^{2}=0.16\right)$ with reduced model $\left(r^{2}=0.08\right)$ yielded a $(1-\beta)$ estimate of 0.72 (Supplementary Fig. S4).

Reproducibility of findings across discovery and validation subsets The stability of estimates linking TNF-KYN/TRP status with clinical variables across discovery and validation sets are shown in Fig. 4c-e. Comparison of AUC across discovery $(n=$ 36) and validation sets $(n=36)$ for the association between TNF-KYN/TRP groups and IDS-SR scores (0.73 vs. $0.77, \chi^{2}(1)=$ $0.11, p=0.74)$, anhedonia (0.71 vs. $\left.0.77, X^{2}(1)=0.15, p=0.69\right)$, and MGH-ATRQ scores (0.66 vs. $0.67, X^{2}(1)=0, p=0.98$ ) indicated good stability of estimates and replicability across sample sets.

\section{DISCUSSION}

Robust relationships were found between KP metabolites in the plasma and CSF. Moreover, inflammatory measures in the plasma, especially TNF, were strongly correlated with Plasma-KP. Path analyses indicated directional associations between plasma inflammatory mediators and Plasma-KP that were in turn associated with KP metabolites in the CSF. Plasma TNF was the strongest predictor of Plasma-KP as represented by KYN/TRP and clustering of these variables yielded two subgroups of patients with high and low combinations of plasma TNF and KYN/TRP. High TNF-KYN/TRP status was associated with greater severity of 
A. Linear Fit of Plasma KYN/TRP and CSF KYN/TRP

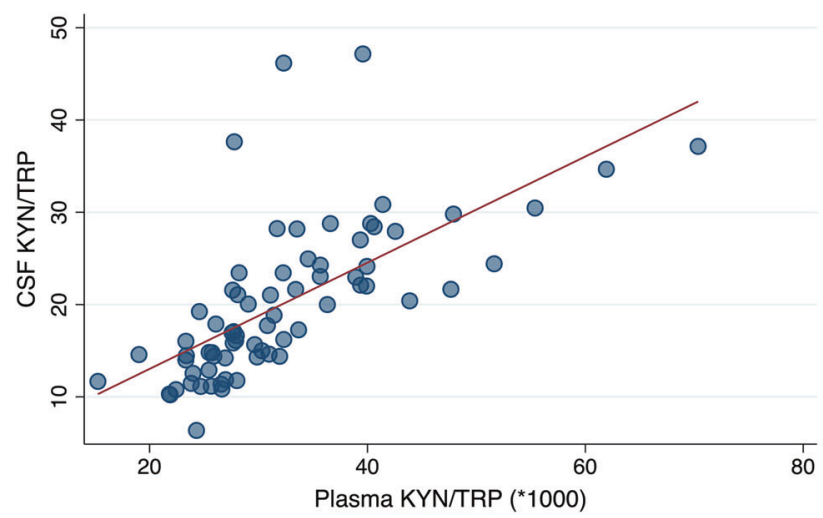

C. Linear Fit of Plasma KYN/TRP and CSF AA

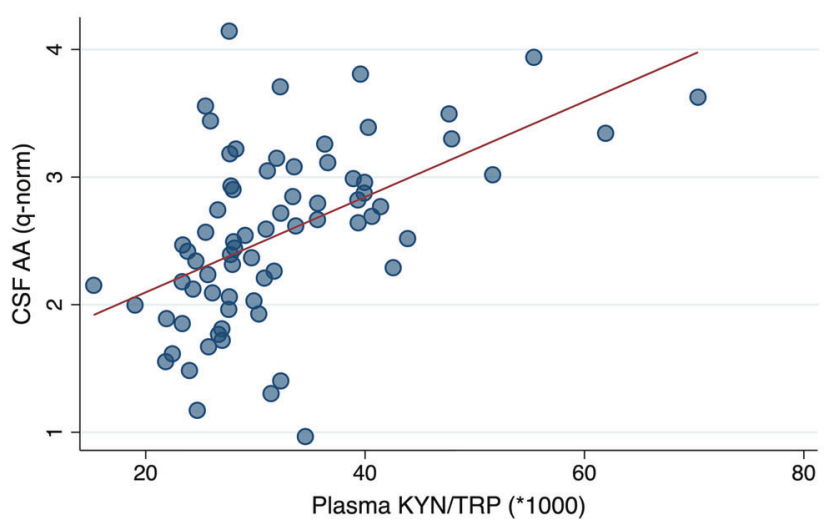

B. Linear Fit of Plasma KYN/TRP and CSF KYN

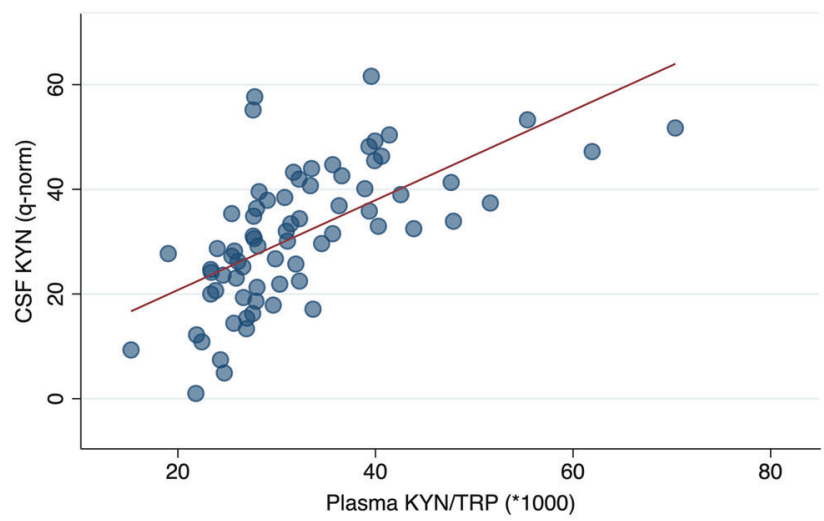

D. LInear Fit of Plasma KYN/TRP and CSF QA

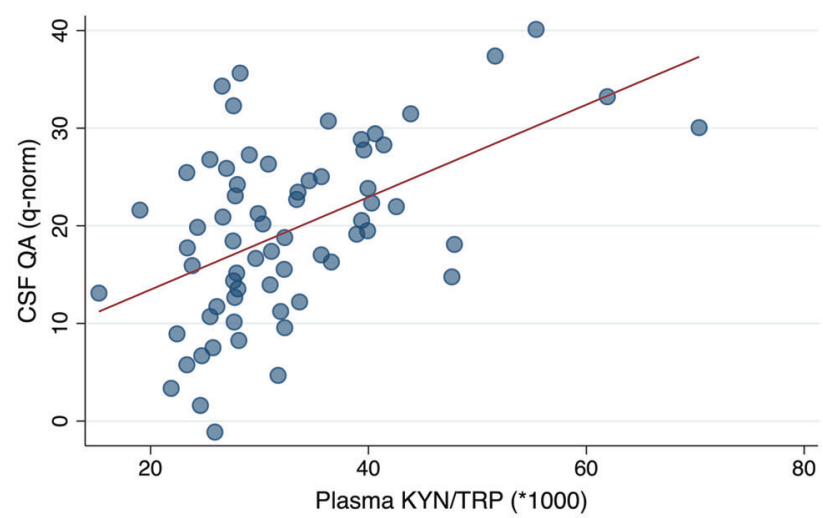

Fig. 2 Bivariate associations between Plasma KYN/TRP and CSF kynurenine pathway metabolites. The panel presents linear fits between individual immune and kynurenine pathway markers in the plasma and CSF. KYN/TRP is represented as a proxy for indoleamine-2dioxygenase (IDO) activity, as it is responsible for generating kynurenine from tryptophan. a Demonstrates significant linear association between plasma kynurenine/tryptophan ratio (KYN/TRP, ${ }^{*} 1000$ for graphic scaling) and CSF KYN/TRP $[r(99 \% \mathrm{Cl})=0.77(0.50-0.90)]$. Similar relationships are demonstrated in (b) between plasma KYN/TRP and CSF kynurenine $[\mathrm{KYN}, r(99 \% \mathrm{Cl})=0.72(0.42-0.88)]$; $\mathbf{c}$ between plasma $\mathrm{KYN} / \mathrm{TRP}$ and CSF anthranilic acid $[\mathrm{AA}, r(99 \% \mathrm{Cl})=0.60(0.22-0.82)]$, and $\mathbf{d}$ between plasma $\mathrm{KYN} / \mathrm{TRP}$ and CSF quinolinic acid $[\mathrm{QA}, r(99 \% \mathrm{CI})=$ $0.52(0.08-0.79)]$. All $p$-values were $<0.0001$.

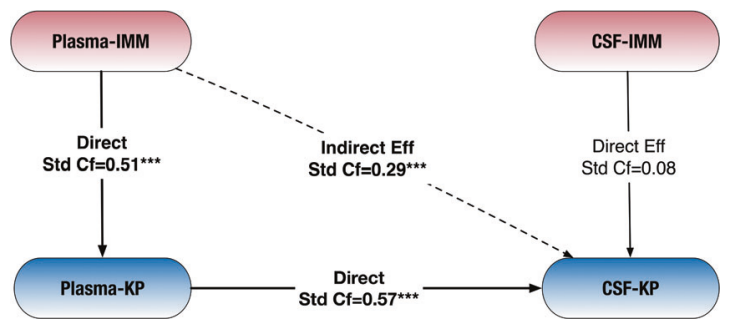

Fig. 3 Path diagram of the directional associations between plasma and CSF immune markers (IMM) and kynurenine pathway (KP) metabolites. Path analysis was conducted to test the directionality of relationships among the extracted component factors controlling for age, race, and BMI (as detailed in the text). The broken arrows represent indirect effects and the filled arrows represent direct effects with increasing line widths signifying stronger effects. Although included in the analyses, covariates are excluded from this figure to improve clarity. Analytic details are provided in Supplementary Table S6A-D. Abbreviations: Plasma-IMM plasma immune component factor, Plasma-KP plasma kynurenine pathway component factor, CSF-IMM cerebrospinal immune component factor, CSF-KP cerebrospinal kynurenine pathway component factor, Std $\mathrm{Cf}$ standardized path coefficients (calculated by normalizing to variance). *Significant effects at $p<0.001$. depression, anhedonia, and treatment nonresponse, possibly reflecting a unique biological phenotype that may be especially responsive to treatment targeting inflammation, the $\mathrm{KP}$, or its downstream effects on glutamate receptors.

The results presented herein represent the most comprehensive assessment of KP metabolites and inflammatory markers, and their interactions in patients with depression. The findings are remarkably consistent with our previously published data demonstrating strong relationships between concentrations of KP metabolites in blood and CSF of patients receiving IFN-a [14] and with other studies reporting similar associations among individuals with other neuropsychiatric disorders [53-55]. Furthermore, these data indicate that plasma KYN/TRP is an excellent proxy of the KP in the CSF of depressed patients-a conclusion supported by our path analysis indicating that CSF-KP concentrations are strongly associated with KP metabolite concentrations in the plasma. Also consistent with our findings with IFN- $a$, path analysis indicated that peripheral KP metabolites were robustly impacted by peripheral immune activation, especially as represented by TNF. Previous literature has demonstrated the capacity of TNF to stimulate IDO both in vitro and in vivo, including the blockade of LPS-induced IDO in the periphery by the TNF antagonist etanercept, which does not cross the blood-brain barrier (BBB) $[3,56,57]$. However, this is the first study in depressed patients to support the directionality of the pathway 


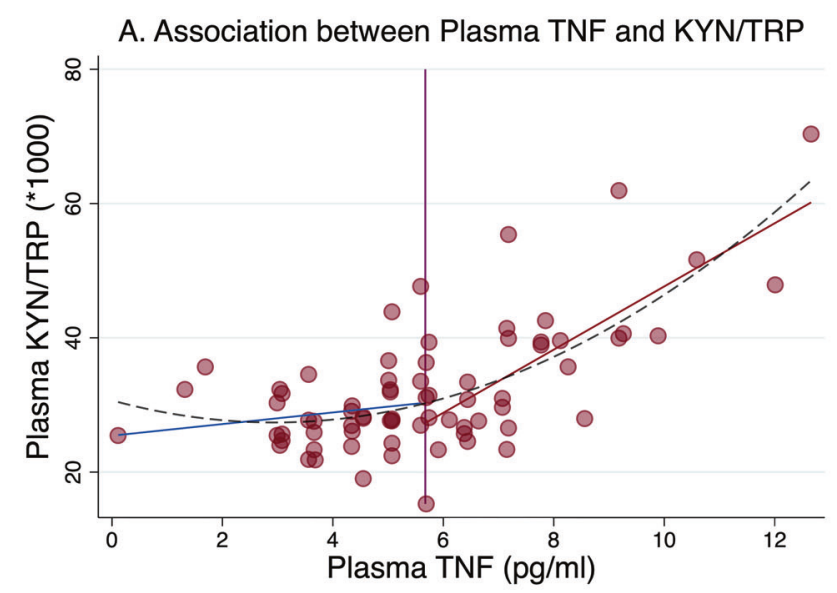

B. K-means Clustering of Plamsa TNF and KYN/TRP

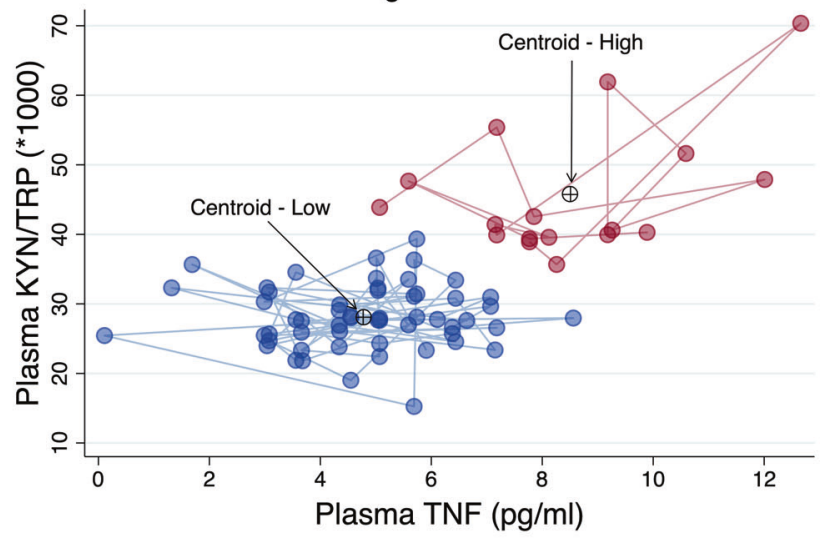

C. ROC-Curve of TNF-KYN/TRP versus IDS-SR
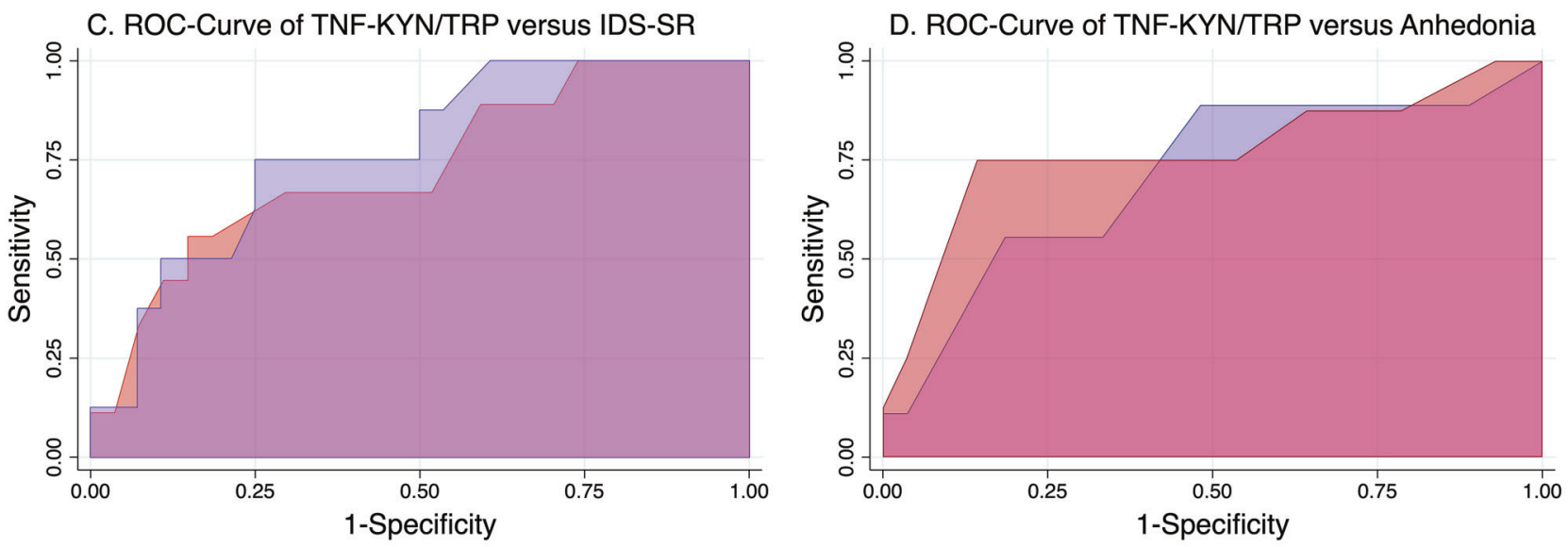

E. ROC-Curve of TNF-KYN/TRP versus MGH-ATRQ
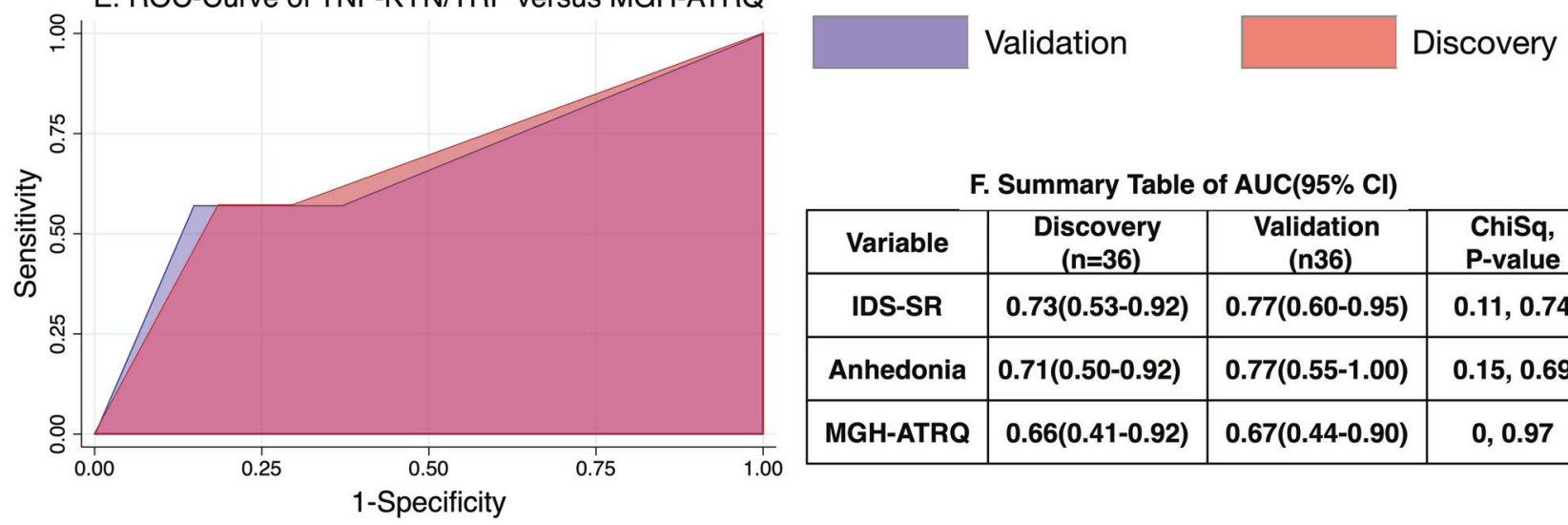

F. Summary Table of AUC(95\% Cl)

\begin{tabular}{|c|c|c|c|}
\hline Variable & $\begin{array}{c}\text { Discovery } \\
(\mathrm{n}=36)\end{array}$ & $\begin{array}{c}\text { Validation } \\
(\mathrm{n} 36)\end{array}$ & $\begin{array}{c}\text { ChiSq, } \\
\text { P-value }\end{array}$ \\
\hline IDS-SR & $0.73(0.53-0.92)$ & $0.77(0.60-0.95)$ & $0.11,0.74$ \\
\hline Anhedonia & $0.71(0.50-0.92)$ & $0.77(0.55-1.00)$ & $0.15,0.69$ \\
\hline MGH-ATRQ & $0.66(0.41-0.92)$ & $0.67(0.44-0.90)$ & $0,0.97$ \\
\hline
\end{tabular}

Fig. 4 Clustering patients based on plasma TNF and KYN/TRP to associate with clinical characteristics. a Demonstrates nonlinear, quadratic fit between plasma tumor necrosis factor (TNF) and kynurenine/tryptophan (KYN/TRP, ${ }^{*} 1000$ for graphic scaling). The curved (quadratic/polynomial) fit (broken line) was significantly stronger than the linear fit (linear/nonlinear slope difference $[\mathrm{F}(1,69)=11.3, p=$ 0.001]. The blue line shows the linear fit between Plasma KYN/TRP and Plasma TNF $<5.67 \mathrm{pg} / \mathrm{ml}$, whereas the red line shows the same fit at Plasma TNF $>5.67 \mathrm{pg} / \mathrm{ml}$. b Demonstrates the use of $K$-means clustering to subgroup patients into Low $(n=55)$ and High $(n=17)$ TNF-KYN/ TRP groups. A connected scatterplot associating plasma TNF in the $x$-axis and KYN/TRP $\left({ }^{*} 1000\right)$ in $y$-axes demonstrates significant separation between clusters. The High and Low TNF-KYN/TRP groups are represented using the filled red and blue circles, respectively. The cross-haired, hollow circles in the center of the groups represent the centroid values for the two clusters. c-f Present area under the curve estimates (AUC) obtained from receiver operating (ROC) curves to compare the stability of the association between TNF-KYN/TRP clusters and clinical variables across the "Discovery" (shaded purple/blue) and "Validation" (shaded orange) sets. ROC curves associating TNF-KYN/TRP group status (High vs. Low) with depression severity (IDS-SR scores) is presented in (c); with anhedonia (anhedonia subscale scores) in (d); and treatment nonresponse (MGH-ATRQ scores) in (e). f Presents comparison of AUC measures across Discovery and Validation sets. The insignificant differences in AUC between the randomly split Discovery and Validation subset, indicates satisfactory cross-validation. 
linking peripheral inflammatory markers to peripheral KP metabolites extending to the KP metabolites in the CNS. This pathway has been well-described in laboratory animals; most definitively in a recent study in mice where activation of the central KP following immune stimulation by LPS could be reversed by overloading LAT1 with leucine, thereby limiting access of peripheral KYN to the brain and abrogating depressive-like behavior [25]. It should be noted that significant correlations were also found between plasma and CSF QA, as well as AA. AA is believed to passively diffuse into the CNS, whereas $Q A$ is not believed to readily cross the BBB [58]. Indeed, although brain QA is known to originate from local sources, its formation by brain tissue increases in conditions of both systemic and brain immune activation [59]. Of note, significant correlations between plasma and CSF QA were also found in our studies of IFN-a-treated patients and has been found in patients with HIV $[14,16]$. One possibility is that increased peripheral inflammation may disrupt BBB integrity or impact LAT-1 activity $[11,16]$. Nevertheless, the results of our path model along with studies blocking KYN transport to the brain in rodents support the notion that peripheral KYN serves as the primary substrate for KP metabolites in the brain [11, 58], with some contribution from downstream peripheral metabolites including 3HKYN, which is also transported into the brain by LAT-1 [58].

Previous studies have related KP metabolites to overall depression severity including a relationship with anhedonia/reduced motivation and volume loss in the striatum [14, 16-19,60]. Our data are in support of these findings and suggest that the combined activation of inflammation and KP in the periphery may represent a unique subtype of patients that may more powerfully map onto previous associations between symptoms of depression including anhedonia and KP metabolites alone. Moreover, our data have additionally mapped this biological subtype to treatment resistance possibly related to the combined effects of inflammation and KP metabolites on treatment response [23, 30, 32, 61-63].

Regarding treatment of depressed patients with inflammatory activation of the KP, several IDO inhibitors are currently under development (for treatment of cancer) and may be crosspurposed for treatment of depression $[2,16]$. Moreover, blocking KYN transport across the BBB using leucine-a competitive LAT-1inhibitor-may also be warranted, especially given the success in preclinical models [25]. Preclinical data also suggest that physical exercise/activity may protect against stress-induced depression by increasing expression of muscle enzymes that convert KYN into KYNA resulting in decreased peripheral KYN available to cross the BBB into the brain [64]. Finally, glutamate receptor modulating agents such as ketamine, memantine, and riluzole may be relevant for modulating the downstream effects of KP metabolites on glutamate neurotransmission $[10,61]$.

The strengths of the study include the use of a wellcharacterized group of medically stable, unmedicated depressed subjects; measurement of inflammatory and KP markers in the plasma and CSF using standardized collection and assay procedures; and utilization of path modeling and machine-based learning approaches to profile the effects of combined dysregulation of inflammation and KP metabolites upon depressive symptoms, anhedonia, and treatment non-responsiveness. However, several caveats should be considered. A control group was not used, because the primary focus of this study was to examine the relationships among immune and $\mathrm{KP}$ metabolites in the plasma and CSF within depressed patients, leverage this information to define subgroups of depressed subjects with/without combined activation of these pathways, and to test their relevance to specific symptom clusters of depression. Nevertheless, the plasma-CSF associations identified in this study are remarkably similar to those reported in nondepressed medically ill and healthy control subjects in past human studies [14]. This data is also in line with a large volume of preclinical studies and limited data from healthy volunteers undergoing inflammatory challenge $[7,8,65]$. Another limitation is that we had no measure of IFN- $\gamma$, which has been shown to be associated with Plasma-KP [66]. Suicidal behavior could not be analyzed due to previously mentioned reasons [19-21]. Besides, given the paucity of specific scales to measure anhedonia in mood disordered subjects, we used a subset of items extracted from items from the IDS-SR scale. To minimize selection bias, 4/5 items from an anhedonic cluster previously identified by a principal component analysis were combined based on the best Cronbach's a (0.73). Finally, the data included in the study were cross-sectional in nature and hence estimations of directionality are restricted to extrapolations from a path model, and no claims of causal associations can be made.

In conclusion, this study characterizes a peripheral pathway involving inflammatory mediators and KP metabolites that drives the KP in the brain. In addition, a subgroup of depressed patients with combined increases of plasma inflammatory and KP measures exhibited increases in depressive symptom severity, anhedonia, and treatment non-responsiveness. Targeting of inflammation, IDO, KYN transport into the brain, and/or glutamate dysregulation in this biological subgroup of major depressions will support precision medicine and potentially improve treatment response.

\section{FUNDING AND DISCLOSURE}

This study was supported by grants R01MH087604 (AHM), R01MH107033 (EH), K23MH091254 (EH), R01MH112076 (AHM, EH), $F(J C F)$ from the National Institute of Mental Health and by the National Center for Advancing Translational Sciences of the National Institutes of Health under Award Number UL1TR002378 (Georgia Clinical and Translational Alliance, Georgia CTSA). The content is solely the responsibility of the authors and does not necessarily represent the official views of the National Institutes of Health. The funding sources had no role in the design and conduct of the study; collection, management, analysis, and interpretation of the data; preparation, review, or approval of the manuscript; and decision to submit the manuscript for publication. All authors have no conflict of interest or financial relationships to disclose.

\section{ACKNOWLEDGEMENTS}

We acknowledge the support provided by the staff of Georgia Clinical \& Translational Science Alliance (Georgia CTSA).

\section{AUTHOR CONTRIBUTIONS}

EH performed all computations and prepared all aspects of the manuscript. AHM conceived and executed the parent project and participated fully in the writing of this manuscript. JCF provided lab support, authentication, and quality control of the biological assays in addition to writing support. TP conducted lumbar punctures. BJW conducted structured clinical assessments and scale ratings. JRW participated in preliminary analysis, and DRG and WB assisted in writing of this manuscript.

\section{ADDITIONAL INFORMATION}

Supplementary Information accompanies this paper at (https://doi.org/10.1038/ s41386-020-0607-1).

Publisher's note Springer Nature remains neutral with regard to jurisdictional claims in published maps and institutional affiliations.

\section{REFERENCES}

1. Dantzer R. Role of the kynurenine metabolism pathway in inflammation-induced depression: preclinical approaches. Curr Top Behav Neurosci. 2017;31:117-38.

2. Schwarcz R. The kynurenine pathway of tryptophan degradation as a drug target. Curr Opin Pharmacol. 2004:4:12-7. 
3. O'Connor JC, Andre C, Wang Y, Lawson MA, Szegedi SS, Lestage J, et al. Interferon-gamma and tumor necrosis factor-alpha mediate the upregulation of indoleamine 2,3-dioxygenase and the induction of depressive-like behavior in mice in response to bacillus Calmette-Guerin. J Neurosci 2009;29:4200-9.

4. Guillemin GJ, Kerr SJ, Smythe GA, Smith DG, Kapoor V, Armati PJ, et al. Kynurenine pathway metabolism in human astrocytes: a paradox for neuronal protection. J Neurochem. 2001;78:842-53.

5. Du F, Schmidt W, Okuno E, Kido R, Kohler C, Schwarcz R. Localization of kynurenine aminotransferase immunoreactivity in the rat hippocampus. J Comp Neurol. 1992;321:477-87.

6. Wejksza K, Rzeski W, Okuno E, Kandefer-Szerszen M, Albrecht J, Turski WA. Demonstration of kynurenine aminotransferases I and II and characterization of kynurenic acid synthesis in oligodendrocyte cell line (OLN-93). Neurochem Res. 2005;30:963-8

7. Schwarcz R, Bruno JP, Muchowski PJ, Wu HQ. Kynurenines in the mammalian brain: when physiology meets pathology. Nat Rev Neurosci. 2012;13:465-77.

8. Dantzer R, O'Connor JC, Lawson MA, Kelley KW. Inflammation-associated depression: from serotonin to kynurenine. Psychoneuroendocrinology 2011;36: 426-36.

9. Guillemin GJ. Quinolinic acid, the inescapable neurotoxin. Febs J. 2012;279: 1356-65.

10. Schwarcz R. Kynurenines and glutamate: multiple links and therapeutic implications. Adv Pharmacol. 2016;76:13-37.

11. Dantzer R, Walker AK. Is there a role for glutamate-mediated excitotoxicity in inflammation-induced depression? J Neural Transm (Vienna) 2014;121:925-32.

12. O'Connor JC, Lawson MA, Andre C, Briley EM, Szegedi SS, Lestage J, et al. Induction of IDO by bacille Calmette-Guerin is responsible for development of murine depressive-like behavior. J Immunol. 2009;182:3202-12.

13. Walker AK, Budac DP, Bisulco S, Lee AW, Smith RA, Beenders B, et al. NMDA receptor blockade by ketamine abrogates lipopolysaccharide-induced depressivelike behavior in C57BL/6J mice. Neuropsychopharmacology 2013;38:1609-16.

14. Raison CL, Dantzer R, Kelley KW, Lawson MA, Woolwine BJ, Vogt G, et al. CSF concentrations of brain tryptophan and kynurenines during immune stimulation with IFN-alpha: relationship to CNS immune responses and depression. Mol Psychiatry 2010;15:393-403.

15. Capuron L, Neurauter G, Musselman DL, Lawson DH, Nemeroff CB, Fuchs D, et al. Interferon-alpha-induced changes in tryptophan metabolism. Relationship to depression and paroxetine treatment. Biol Psychiatry 2003;54:906-14.

16. Savitz J. The kynurenine pathway: a finger in every pie. Mol Psychiatry 2020; 25:131-47.

17. Savitz J, Dantzer R, Meier TB, Wurfel BE, Victor TA, Mclntosh SA, et al. Activation of the kynurenine pathway is associated with striatal volume in major depressive disorder. Psychoneuroendocrinology 2015;62:54-8.

18. Capuron L, Schroecksnadel S, Feart C, Aubert A, Higueret D, Barberger-Gateau P, et al. Chronic low-grade inflammation in elderly persons is associated with altered tryptophan and tyrosine metabolism: role in neuropsychiatric symptoms. Biol Psychiatry 2011;70:175-82.

19. Bay-Richter C, Linderholm KR, Lim CK, Samuelsson M, Traskman-Bendz L, Guillemin GJ, et al. A role for inflammatory metabolites as modulators of the glutamate $\mathrm{N}$-methyl-D-aspartate receptor in depression and suicidality. Brain Behav Immun. 2015:43:110-7.

20. Erhardt $S$, Lim CK, Linderholm KR, Janelidze S, Lindqvist $D$, Samuelsson $M$, et al. Connecting inflammation with glutamate agonism in suicidality. Neuropsychopharmacology 2013;38:743-52.

21. Bryleva EY, Brundin L. Kynurenine pathway metabolites and suicidality. Neuropharmacology 2017;112(Pt B):324-30.

22. Haroon E, Daguanno AW, Woolwine BJ, Goldsmith DR, Baer WM, Wommack EC, et al. Antidepressant treatment resistance is associated with increased inflammatory markers in patients with major depressive disorder. Psychoneuroendocrinology 2018;95:43-9.

23. Moaddel R, Shardell M, Khadeer M, Lovett J, Kadriu B, Ravichandran $S$, et al. Plasma metabolomic profiling of a ketamine and placebo crossover trial of major depressive disorder and healthy control subjects. Psychopharmacology (Berl). 2018:235:3017-30.

24. Parrott JM, Redus L, Santana-Coelho D, Morales J, Gao X, O'Connor JC. Neurotoxic kynurenine metabolism is increased in the dorsal hippocampus and drives distinct depressive behaviors during inflammation. Transl Psychiatry 2016;6:e918.

25. Walker AK, Wing EE, Banks WA, Dantzer R. Leucine competes with kynurenine for blood-to-brain transport and prevents lipopolysaccharide-induced depressionlike behavior in mice. Mol Psychiatry. 2019;24:1523-32.

26. First MB, Spitzer RL, Gibbon M, Williams JBW. Structured Clinical Interview for DSM-IV-TR Axis I Disorders, Research Version. (SCID-IV) (New York: Biometrics Research, New York State Psychiatric Institute, 2002).

27. Hamilton M. A rating scale for depression. J Neurol Neurosurg Psychiatry. 1960;23:56-62.
28. Baumeister D, Ciufolini S, Mondelli V. Effects of psychotropic drugs on inflammation: consequence or mediator of therapeutic effects in psychiatric treatment? Psychopharmacology (Berl). 2016;233:1575-89.

29. Felger JC, Li Z, Haroon E, Woolwine BJ, Jung MY, Hu X, et al. Inflammation is associated with decreased functional connectivity within corticostriatal reward circuitry in depression. Mol Psychiatry 2016;21:1358-65.

30. Haroon E, Fleischer CC, Felger JC, Chen X, Woolwine BJ, Patel T, et al. Conceptual convergence: increased inflammation is associated with increased basal ganglia glutamate in patients with major depression. Mol Psychiatry 2016;21:1351-7.

31. Felger JC, Haroon E, Patel TA, Goldsmith DR, Wommack EC, Woolwine BJ, et al. What does plasma CRP tell us about peripheral and central inflammation in depression? Mol Psychiatry. 2018.

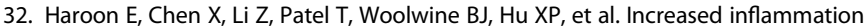
and brain glutamate define a subtype of depression with decreased regional homogeneity, impaired network integrity, and anhedonia. Transl Psychiatry 2018;8:189.

33. Trivedi $M H$, Rush AJ, Ibrahim HM, Carmody TJ, Biggs MM, Suppes $T$, et al. The Inventory of Depressive Symptomatology, Clinician Rating (IDS-C) and Self-Report (IDS-SR), and the Quick Inventory of Depressive Symptomatology, Clinician Rating (QIDS-C) and Self-Report (QIDS-SR) in public sector patients with mood disorders: a psychometric evaluation. Psychol Med. 2004;34:73-82.

34. Ameli R, Luckenbaugh DA, Gould NF, Holmes MK, Lally N, Ballard ED, et al. SHAPSC: the Snaith-Hamilton pleasure scale modified for clinician administration. PeerJ 2014;2:e429.

35. Chandler GM, losifescu DV, Pollack MH, Targum SD, Fava M. Reasearch: validation of the Massachusetts General Hospital Antidepressant Treatment History Questionnaire (ATRQ). CNS Neurosci Ther. 2010;16:322-5.

36. Berman RM, Marcus RN, Swanink R, McQuade RD, Carson WH, Corey-Lisle PK, et al. The efficacy and safety of aripiprazole as adjunctive therapy in major depressive disorder: a multicenter, randomized, double-blind, placebo-controlled study. J Clin Psychiatry. 2007;68:843-53.

37. Gibson TB, Jing Y, Smith Carls G, Kim E, Bagalman JE, Burton WN, et al. Cost burden of treatment resistance in patients with depression. Am J Manag Care. 2010;16:370-7.

38. Marcus RN, McQuade RD, Carson WH, Hennicken D, Fava M, Simon JS, et al. The efficacy and safety of aripiprazole as adjunctive therapy in major depressive disorder: a second multicenter, randomized, double-blind, placebo-controlled study. J Clin Psychopharmacol. 2008;28:156-65.

39. Bolstad BM, Irizarry RA, Astrand M, Speed TP. A comparison of normalization methods for high density oligonucleotide array data based on variance and bias. Bioinformatics 2003;19:185-93.

40. Repsilber D, Kern S, Telaar A, Walzl G, Black GF, Selbig J, et al. Biomarker discovery in heterogeneous tissue samples -taking the in-silico deconfounding approach. BMC Bioinformatics 2010;11:27.

41. Shapiro SS, Wilk MB. An analysis of variance test for normality (complete samples). Biometrika 1965;52:591-611.

42. Mueser KT, Kim M, Addington J, McGurk SR, Pratt SI, Addington DE. Confirmatory factor analysis of the quality of life scale and new proposed factor structure for the quality of life scale-revised. Schizophr Res. 2017;181:117-23.

43. Perry JL, Nicholls AR, Clough PJ, Crust L. Assessing model fit: caveats and recommendations for confirmatory factor analysis and exploratory structural equation modeling. Meas Phys Educ Exerc Sci. 2015;19:12-21.

44. Schreiber JB, Nora A, Stage FK, Barlow EA, King J. Reporting structural equation modeling and confirmatory factor analysis results: a review. Reporting. J Educ Res. 2006;99:323-37.

45. Cha J, Greenberg T, Song I, Blair Simpson H, Posner J, Mujica-Parodi LR. Abnormal hippocampal structure and function in clinical anxiety and comorbid depression. Hippocampus 2016;26:545-53.

46. Acock AC. Discovering Structural Equation Modeling Using Stata. Revised Edition. 2 edn. (College Station, TX, USA: Stata Press, 2013).

47. Tibshirani R. Regression shrinkage and selection via the lasso: a retrospective. J R Stat Soc Ser B (Stat Methodol). 2011;73:273-82.

48. Guan Y, Stephens M. Bayesian variable selection regression for genome-wide association studies and other large-scale problems. Ann Appl Stat. 2011;5: 1780-815

49. Kiecolt-Glaser JK, Derry HM, Fagundes CP. Inflammation: depression fans the flames and feasts on the heat. Am J Psychiatry. 2015;172:1075-91.

50. Franceschi C, Campisi J. Chronic inflammation (inflammaging) and its potential contribution to age-associated diseases. J Gerontol Ser A Biol Sci Med Sci. 2014;69(Suppl 1):S4-9.

51. Sabia S, Kivimaki M, Shipley MJ, Marmot MG, Singh-Manoux A. Body mass index over the adult life course and cognition in late midlife: the Whitehall II Cohort Study. Am J Clin Nutr. 2009;89:601-7.

52. Kraemer HC, Kupfer DJ. Size of treatment effects and their importance to clinical research and practice. Biol Psychiatry 2006;59:990-6. 
53. Jacobs KR, Lim CK, Blennow K, Zetterberg $H$, Chatterjee $P$, Martins RN, et al. Correlation between plasma and CSF concentrations of kynurenine pathway metabolites in Alzheimer's disease and relationship to amyloid-beta and tau. Neurobiol Aging 2019;80:11-20.

54. Sellgren CM, Gracias J, Jungholm O, Perlis RH, Engberg G, Schwieler L, et al. Peripheral and central levels of kynurenic acid in bipolar disorder subjects and healthy controls. Transl Psychiatry 2019;9:37.

55. Hestad KA, Engedal K, Whist JE, Farup PG. The Relationships among tryptophan, kynurenine, indoleamine 2,3-dioxygenase, depression, and neuropsychological performance. Front Psychol. 2017;8:1561.

56. Robinson CM, Shirey KA, Carlin JM. Synergistic transcriptional activation of indoleamine dioxygenase by IFN-gamma and tumor necrosis factor-alpha. J Interferon Cytokine Res. 2003;23:413-21.

57. Robinson CM, Hale PT, Carlin JM. The role of IFN-gamma and TNF-alpharesponsive regulatory elements in the synergistic induction of indoleamine dioxygenase. J Interferon Cytokine Res. 2005;25:20-30.

58. Fukui S, Schwarcz R, Rapoport SI, Takada Y, Smith QR. Blood-brain barrie transport of kynurenines: implications for brain synthesis and metabolism. J Neurochem. 1991;56:2007-17.

59. Heyes MP, Morrison PF. Quantification of local de novo synthesis versus blood contributions to quinolinic acid concentrations in brain and systemic tissues. J Neurochem. 1997:68:280-8.
60. Cho HJ, Savitz J, Dantzer R, Teague TK, Drevets WC, Irwin MR. Sleep disturbance and kynurenine metabolism in depression. J Psychosom Res. 2017;99:1-7.

61. Haroon $E$, Miller AH. Inflammation effects on brain glutamate in depression mechanistic considerations and treatment implications. Curr Top Behav Neurosci. 2017;31:173-98.

62. Haroon E, Miller AH, Sanacora G. Inflammation, glutamate, and glia: a trio of trouble in mood disorders. Neuropsychopharmacology 2017;42:193-215.

63. Haroon E, Woolwine BJ, Chen X, Pace TW, Parekh S, Spivey JR, et al. IFN-alpha-induced cortical and subcortical glutamate changes assessed by magnetic resonance spectroscopy. Neuropsychopharmacology 2014;39: 1777-85.

64. Agudelo LZ, Femenia T, Orhan F, Porsmyr-Palmertz M, Goiny M, MartinezRedondo $V$, et al. Skeletal muscle PGC-1alpha1 modulates kynurenine metabolism and mediates resilience to stress-induced depression. Cell 2014 159:33-45.

65. Kruse JL, Cho JH, Olmstead R, Hwang L, Faull K, Eisenberger NI, et al. Kynurenine metabolism and inflammation-induced depressed mood: a human experimental study. Psychoneuroendocrinology 2019;109:104371.

66. Buntinx M, Moreels M, Vandenabeele F, Lambrichts I, Raus J, Steels $\mathrm{P}$, et al. Cytokine-induced cell death in human oligodendroglial cell lines: I. Synergistic effects of IFN-gamma and TNF-alpha on apoptosis. J Neurosci Res. 2004;76: 834-45. 